\title{
Appropriate Vancomycin Use and Incidence of Vancomycin-Resistant Enterococci in Liver Transplant Recipients
}

\author{
Jessica Ferguson ${ }^{1,}{ }^{*}$, Marisa Holubar ${ }^{1}$, Waldo Concepcion ${ }^{2}$, Dora Y. Ho ${ }^{1}$
}

1. Division of Infectious Diseases and Geographic Medicine, Department of Medicine, Stanford, CA, USA; E-Mails: jferg1@stanford.edu; mholubar@stanford.edu; jsbach@stanford.edu

2. Division of Abdominal Transplantation, Department of Surgery, Stanford University School of Medicine, Stanford, CA, USA; E-Mails: waldo1@stanford.edu

* Correspondence: Jessica Ferguson; E-Mail: jferg1@stanford.edu

Academic Editor: Haval Shirwan

OBM Transplantation

2020, volume 4, issue 4

doi:10.21926/obm.transplant.2004122
Received: July 26, 2020

Accepted: October 28, 2020

Published: November 09, 2020

\begin{abstract}
Liver transplant recipients (LTRs) are at risk for vancomycin-resistant Enterococcus (VRE) infections which can lead to significant morbidity or mortality. Antibiotic exposure, including vancomycin, is associated with greater risk of VRE infection. This study aimed to assess the appropriateness of vancomycin use and rates of VRE infection in this population. We performed a retrospective cohort study of 327 patients who underwent liver transplantation at our center from January 1, 2012 to June 30, 2017. Sixty (18.3\%) LTRs had at least one VREpositive culture between six-months pre-transplant and six-months post-transplant. LTRs with VRE had greater vancomycin exposure as compared to VRE-negative LTRs $(p<0.05)$ and were more likely to receive prolonged vancomycin courses ( 72 hours, $p<0.05$ ). Overall appropriateness of vancomycin use $>72$ hours among VRE-positive LTRs was only $26.8 \%$. Inappropriate vancomycin use most commonly occurred in patients with presumed sepsis without an identifiable source or pneumonia with negative respiratory cultures. Our findings illustrate an opportunity to improve antibiotic stewardship and reduce vancomycin use in the transplant population.
\end{abstract}

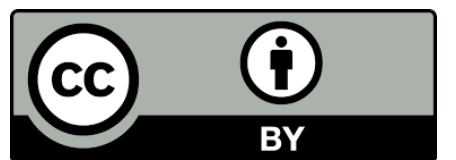

(C) 2020 by the author. This is an open access article distributed under the conditions of the Creative Commons by Attribution License, which permits unrestricted use, distribution, and reproduction in any medium or format, provided the original work is correctly cited. 


\section{Keywords}

Vancomycin-resistant enterococcus; liver transplant; antimicrobial stewardship; vancomycin

\section{Introduction}

Bacterial infections are associated with significant morbidity and mortality in patients with endstage liver disease (ESLD) [1]. In particular, ESLD patients are at greater risk for infections with multidrug resistant bacteria due to their underlying illness and comorbidities, frequent hospitalizations, prior exposure to antibiotics and disruption of normal gastrointestinal flora [2, 3]. Among the multidrug resistant bacteria, vancomycin-resistant Enterococcus (VRE) infections have been associated with increased overall mortality in liver transplant recipients (LTRs) [4]. Risk factors that lead to greater rates of VRE infections in LTRs include prolonged hospital or ICU courses, biliary tract complications, duration of organ ischemia and prior antibiotic exposure [5,6]. Previous studies have identified exposure to vancomycin, cephalosporins, carbapenems and anti-anaerobic antibiotics as risk factors for the development of VRE colonization and infections $[7,8]$.

Given overall rising rates of antimicrobial resistance, there is an increasing focus worldwide on antibiotic stewardship. Robust antibiotic stewardship, in particular, appropriate de-escalation of antibiotic therapy, will likely have significant positive impacts on the transplant populations [9]. No studies to date have specifically investigated vancomycin exposure in association with VRE infection in LTRs. We chose to focus on VRE infections given high incidence rates at our institution. This study aimed to evaluate the association between total vancomycin exposure and subsequent rates of VRE infection in LTRs, as well as the appropriateness of vancomycin use in this patient population.

\section{Methods}

This is a retrospective cohort study of 327 LTRs transplanted at a single tertiary center (Stanford Hospital) between January 1, 2012 and June 30, 2017. This study was approved by the Institutional Review Board at Stanford University. Inclusion criteria were defined as all adult (age $\geq 18$ years) LTRs who received a liver transplant at Stanford Hospital during the study period, including 30 with multi-organ transplants. LTRs transplanted at outside institutions were excluded.

Chart review was performed to identify positive VRE cultures (including blood, urine, intraabdominal fluid or other sources) among the LTRs from six-months pre-transplant until six-months post-transplant. Any cultures collected with the purpose of screening for VRE colonization were excluded. Culture data were collected from microbiology results for source, date, organisms and susceptibilities. Microbiology results from outside institutions were excluded due to incomplete accessibility to comprehensive results. Data collected also included age, gender, mortality at oneyear post transplant, etiology of liver disease, MELD (Model for End-stage Liver Disease) score at time of transplant and days of vancomycin exposure. The VRE-positive (VRE-pos) group included all LTRs with at least one VRE-positive culture between six-months pre-transplant and six-months posttransplant. Those without any positive cultures for VRE during this period were included in the VREnegative (VRE-neg) group. 
Vancomycin exposure was defined as any administration of intravenous vancomycin at Stanford Hospital from two years prior to transplant until six-months post-transplant. Total vancomycin exposure was calculated as the cumulative number of days in which one or more doses of vancomycin were administered during the defined time period. For patients on hemodialysis, vancomycin exposure represented the cumulative number of days vancomycin was ordered per the electronic medical record to account for non-daily dosing schedules. Outpatient dosing of vancomycin was not included.

Vancomycin use $>72$ hours was classified as appropriate only if its use met one of the following criteria: treatment of methicillin-resistant Staphylococcus aureus (MRSA), coagulase-negative staphylococci (with Mec A PCR positivity), bacterial infections for which vancomycin is the drug of choice, skin and soft tissue infections or other deep-seated infections (e.g. osteomyelitis), for which MRSA infection cannot be ruled out by cultures. Seventy-two hours was chosen as adequate time for culture results to be made available and providers to de-escalate antibiotics based on these results [10]. Mortality rate was defined as death occurring within the first year following liver transplant.

For statistical analysis, continuous variables are reported as means, median and range. Fisher exact test was used to compare characteristics between the two groups. All tests were two-tailed and $P<0.05$ used to determine significance. All analyses were performed using Microsoft Excel and SPSS 24.

\section{Results}

A total of 327 LTRs were identified during the study period. Of these, 60 LTRs had at least one VRE-positive culture (VRE-pos group). The remaining 267 LTRs were included in the VRE-neg group. The demographics of the two groups are shown in Table 1. The VRE-pos group was transplanted at a younger mean age $(54.3$ vs. $57.2, p<0.05)$ and with a significantly higher MELD score (34.5 vs. $22.2, p<0.05)$. The VRE-pos group also included a higher proportion of multi-organ transplant recipients, including 10 LTRs who also received kidney transplants and one liver and heart transplant. Mortality at one-year post-transplant was also greater in the VRE-pos group compared to VRE-neg group ( $23.3 \%$ vs $6.7 \%, p<0.05)$. In the VRE-pos group, the most common reason for transplantation was due to alcoholic cirrhosis and hepatitis C (Table 2). The VRE-neg group had a larger proportion transplanted due to hepatocellular carcinoma (46.1\%), followed by alcoholic cirrhosis and hepatitis C.

Table 1 Demographics of liver transplant recipients (LTRs).

\begin{tabular}{llll}
\hline & VRE-pos $(n=60)$ & VRE-neg $(n=267)$ & P-value \\
\hline Female, no. (\%) & $31(51.7 \%)$ & $78(29.2 \%)$ & $<0.05$ \\
Age at transplant & & & \\
Mean & 54.3 & 57.2 & \\
Median & 57 & 59 & $<0.05$ \\
IQR & $51-60$ & $52-64$ &
\end{tabular}


MELD at transplant

Mean

Median

IQR

Multi-organ transplant

One-year mortality, no. (\%)

\section{5}

37

$33-40$

$11(18.0 \%)$

$14(23.3 \%)$
22.2

19 $<0.05$

11-35

$19(7.0 \%)<0.05$

$18(6.7 \%) \quad<0.05$

Abbreviations: VRE-pos, LTRs with at least one VRE-positive culture; VRE-neg, LTRs without any VRE-positive cultures.

Table 2 Underlying liver disease(s) of the liver transplant recipients (LTRs).

\begin{tabular}{llll}
\hline \multirow{2}{*}{ Underlying Liver Disease } & VRE-Pos $(\mathrm{N}=60)$ & VRE-Neg $(\mathrm{N}=267)$ & P-valve \\
\hline $\mathrm{HCC}$ & $\mathrm{N}(\%)$ & $\mathrm{N}(\%)$ & 0.05 \\
$\mathrm{HCC}+$ additional etiology** & $7(11.7)$ & $17(6.4)$ & $<0.05$ \\
$\mathrm{HCV}$ & $11(18.3)$ & $106(39.7)$ & 0.07 \\
$\mathrm{HCV}+\mathrm{EtOH}$ & $4(6.7)$ & $26(9.8)$ & 0.49 \\
$\mathrm{HBV}$ & $2(3.3)$ & $11(4.1)$ & 1.00 \\
$\mathrm{HBV}+\mathrm{EtOH}$ & 0 & $11(4.1)$ & 1.00 \\
EtOH & $18(30.0)$ & $2(0.8)$ & $<0.05$ \\
Autoimmune & $8(13.3)$ & $42(15.7)$ & $<0.05$ \\
Cryptogenic & $1(1.7)$ & $15(5.6)$ & 1.00 \\
NASH & $3(5.0)$ & $7(2.6)$ & 0.43 \\
Other*** & $6(10.0)$ & $8(3.0)$ & 0.62 \\
\hline
\end{tabular}

** Includes HCV, HBV, alcoholic cirrhosis, autoimmune, cryptogenic, NASH and other

*** Other includes drug and toxin induced liver failure, biliary etiologies and unknown cause of liver failure

There was a total of 67 positive VRE cultures in the VRE-pos group, including 33 urine (49.2\%), 15 blood (22.4\%), 6 peritoneal (9.0\%), 11 with > 1 sites (blood with either urine or peritoneal, 16.4\%) and 2 others (wound or sputum) (3.0\%). The VRE isolates were identified to the species level in 24 cases, including Enterococcus faecium $(n=22)$, Enterococcus faecalis $(n=1)$ and Enterococcus casseliflavus $(n=1)$. Of these 67 positive VRE culture results, $53(79.1 \%)$ resulted in treatment, most commonly with linezolid $(35,66.0 \%)$ or daptomycin $(11,20.8 \%)$. The untreated VRE cultures were all from urine samples $(14,20.9 \%)$. The median time to first VRE-positive culture was within 1 month of transplant.

The VRE-pos LTRs had greater total days of vancomycin exposure (8.32 vs 5.30 days, $<0.05$ ). Extended vancomycin exposure $>72$ hours was also higher in VRE-pos LTRs (9.50 vs 6.71 days, $p<$ 0.05) as compared to VRE-neg LTRs (Table 3). A total of 71 events of vancomycin use $>72$ hours occurred in the VRE-pos group. Of these events, the most common indications cited for vancomycin use included bacteremia/sepsis or systemic inflammatory response syndrome (SIRS) (57.7\%), pneumonia (21.1\%) and intra-abdominal infection (5.6\%) (Table 4). Overall appropriateness of 
vancomycin use $>72$ hours was only $26.8 \%$. Cultures obtained the start of vancomycin led to an identified organism in 38 cases $(n=67,56.7 \%)$. Inappropriate vancomycin use most commonly occurred in "septic" patients without an identifiable infectious source and in patients with pneumonia with negative sputum cultures.

Table 3 Comparison vancomycin use in VRE-pos vs VRE-neg liver transplant recipients (LTRs).

\begin{tabular}{llll}
\hline & VRE-Pos $(\mathrm{N}=60)$ & VRE-Neg $(\mathrm{N}=267)$ & P-value \\
\hline $\begin{array}{l}\text { Cumulative vancomycin use } \\
\text { per LTR, days }\end{array}$ & & & \\
Mean & 8.32 & 5.30 & $<0.05$ \\
Median & 3 & 3 & \\
IQR & $0-15$ & $0-8$ & \\
Vancomycin use & & & $<0.05$ \\
$>72$ hours, days & & & \\
Mean & 9.50 & 6.71 & \\
Median & 7 & 5 & \\
IQR & $5-11$ & $4-8$ & \\
\hline
\end{tabular}

Abbreviations: VRE-pos, LTRs with at least one VRE-positive culture; VRE-neg, LTRs without any VRE-positive cultures.

Table 4 Appropriateness of prolonged ( 72 hours) vancomycin use in VRE-pos liver transplant recipients (LTRs).

\begin{tabular}{lll}
\hline $\begin{array}{l}\text { Suspected infectious source } \\
\text { (per documentation) }\end{array}$ & Number of events $(\mathrm{n}=71)$ & $\begin{array}{l}\text { Events with appropriate } \\
\text { vancomycin use, no. (\%) }\end{array}$ \\
\hline Bacteremia, Sepsis/SIRS & 41 & $10(25.6)$ \\
Intra-abdominal & 4 & $2(50.0)$ \\
Respiratory tract & 15 & $2(13.3)$ \\
Genitourinary & 3 & $2(66.7)$ \\
Skin/Soft Tissue & 3 & $2(66.7)$ \\
Other* & 5 & $1(20.0)$ \\
Total & 71 & $19(26.8)$ \\
\hline
\end{tabular}

Abbreviations: SIRS, systemic inflammatory response syndrome; *Includes: chest tube prophylaxis, surgical prophylaxis, central nervous system (CNS)

\section{Discussion}

The prevalence of VRE-positive cultures among our LTRs was approximately $20 \%$. There were both greater number of cumulative days of vancomycin use and greater incidence of vancomycin use $>72$ hours in VRE-positive LTRs. Based on the higher MELD scores and mortality rate in the VRE- 
pos group, increases in vancomycin use and VRE positivity likely correlate with increased illness severity and healthcare exposure.

When vancomycin was used for $>72$ hours in the VRE-pos group, we found only $26.8 \%$ to have a clinically appropriate reason for its use. Nearly three-quarters of all vancomycin use was not substantiated by microbiologic data. The categories with greatest inappropriate vancomycin use were sepsis with negative culture data and empirical pneumonia coverage with negative respiratory cultures. This highlights a significant opportunity for antibiotic de-escalation based on culture results which has become a prominent strategy for antimicrobial stewardship programs [9]. Additional strategies may be beneficial in cases with negative microbiologic workups [11, 12].

Our study has several limitations. While we focus only on vancomycin exposure, additional antibiotics including fluoroquinolones, beta-lactams and carbapenems have also been associated with the development of VRE infections [7, 8]. Furthermore, we did not assess rates of VRE colonization and all positive VRE-cultures were assumed to represent VRE infections. This assumption may overestimate the true number of VRE infections in this cohort. This study only evaluates overall vancomycin exposure and VRE infections among these patients, but cannot prove direct causality between a particular course of vancomycin and an incidence of VRE infection. Lastly, this study relied on clinician documentation which may not accurately reflect the clinical context leading to antibiotic use and lead to an overestimation of inappropriate vancomycin use.

Future studies are needed to investigate additional variables affecting vancomycin use, including length of hospitalization, number of hospital admissions and illness severity. These factors impact overall antibiotic utilization and the development of resistant organisms independent of vancomycin exposure. Further examination of the practice of antimicrobial use and development of resistant organisms would help develop antibiotic stewardship guidelines, particularly in the transplant populations.

In summary, we found that LTRs with VRE had significantly greater exposure to vancomycin as compared to VRE-negative LTRs. Additionally, up to $73 \%$ of prolonged ( $>72$ hours) vancomycin use was inappropriate, highlighting the need for ongoing improvement in antibiotic stewardship.

\section{Author Contributions}

JF Study concept/design, data collection, analysis of data, drafting of the manuscript; MH Study concept/design, critical revision of the manuscript; WC Critical revision of the manuscript; DH Study concept/design, interpretation of data, drafting and critical revision of the manuscript.

\section{Competing Interests}

The authors have declared that no competing interests exist.

\section{References}

1. Piano S, Brocca A, Mareso S, Angeli P. Infections complicating cirrhosis. Liver Int. 2018; 38: 126133.

2. Patel G, Snydman DR, AST Infectious Diseases Community of Practice. Vancomycin-resistant enterococcus infections in solid organ transplantation. Am J Transplant. 2013; 13: 59-67. 
3. Papanicolaou GA, Meyers BR, Meyers J, Mendelson MH, Lou W, Emre S, et al. Nosocomial infections with vancomycin-resistant enterococcus faecium in liver transplant recipients: Risk factors for acquisition and mortality. Clin Infect Dis Off Publ Infect Dis Soc Am. 1996; 23: 760766.

4. Orloff SL, Busch AM, Olyaei AJ, Corless CL, Benner KG, Flora KD, et al. Vancomycin-resistant enterococcus in liver transplant patients. Am J Surg. 1999; 177: 418-422.

5. McNeil SA, Malani PN, Chenoweth CE, Fontana RJ, Magee JC, Punch JD, et al. Vancomycinresistant enterococcal colonization and infection in liver transplant candidates and recipients: A prospective surveillance study. Clin Infect Dis. 2006; 42: 195-203.

6. Santoro-Lopes G, de Gouvêa EF. Multidrug-resistant bacterial infections after liver transplantation: An ever-growing challenge. World J Gastroenterol. 2014; 20: 6201-6210.

7. Chavers LS, Moser SA, Funkhouser E, Benjamin Jr WH, Chavers P, Stamm AM, et al. Association between antecedent intravenous antimicrobial exposure and isolation of vancomycin-resistant enterococci. Microb Drug Resist. 2003; 9: 69-77.

8. Fridkin SK, Edwards JR, Courval JM, Hill H, Tenover FC, Lawton R, et al. The effect of vancomycin and third-generation cephalosporins on prevalence of vancomycin-resistant enterococci in 126 U.S. adult intensive care units. Ann Intern Med. 2001; 135: 175-183.

9. Zuccaro V, Columpsi P, Sacchi P, Lucà MG, Fagiuoli S, Bruno R. Antibiotic stewardship and empirical antibiotic treatment: How can they get along? Dig Liver Dis. 2017; 49: 579-584.

10. Olson G, Davis AM. Diagnosis and treatment of adults with community-acquired pneumonia. JAMA. 2020; 323: 885-886.

11. Sager R, Kutz A, Mueller B, Schuetz P. Procalcitonin-guided diagnosis and antibiotic stewardship revisited. BMC Med. 2017; 15: 15.

12. Sandkovsky U, Kalil AC, Florescu DF. The use and value of procalcitonin in solid organ transplantation. Clin Transplant. 2015; 29: 689-696.

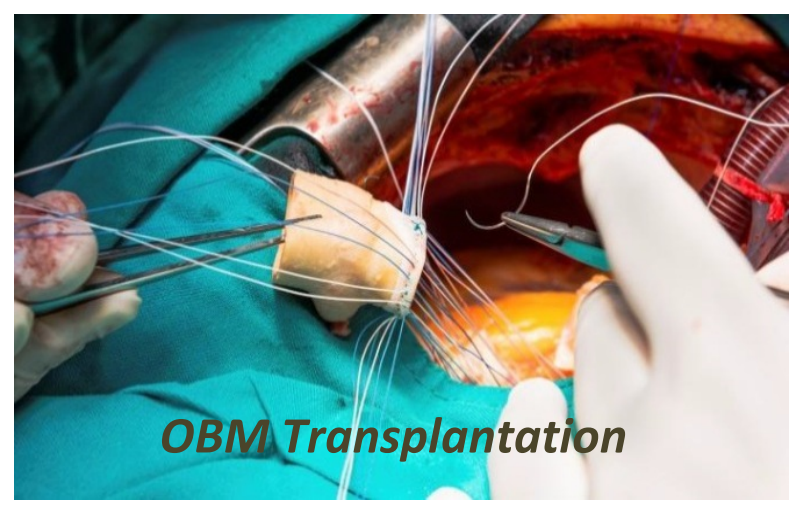

Enjoy OBM Transplantation by:

1. Submitting a manuscript

2. Joining in volunteer reviewer bank

3. Joining Editorial Board

4. Guest editing a special issue

For more details, please visit:

http://www.lidsen.com/journals/transplantation 